\title{
Comparative Antibiotic Sensitivity Pattern of Hospital and Community Acquired Staphylococcus aureus Isolates of Jessore, Bangladesh
}

\author{
Pravas Chandra Roy, Md. Shaheduzzaman, Nigarin Sultana, Iqbal Kabir Jahid* \\ Department of Microbiology, Jessore University of Science and Technology, Jessore, Bangladesh \\ Email: ${ }^{*}$ kjahid@yahoo.com
}

Received 8 August 2015; accepted 6 October 2015; published 9 October 2015

Copyright (C) 2015 by authors and Scientific Research Publishing Inc.

This work is licensed under the Creative Commons Attribution International License (CC BY).

http://creativecommons.org/licenses/by/4.0/

c) (i) Open Access

\begin{abstract}
Staphylococcus aureus has emerged over the past several decades as a leading cause of hospital acquired infections, which are more commonly termed as nosocomial infections. In recent years, strains of this bacterium which are resistant against several types of antibiotics have evolved and their prevalence is becoming a potential epidemiological threat. As there are limited data available on antibiotic resistance patterns of $S$. aureus that are isolated from hospital, the study was undertaken. The study was conducted by collecting swab samples from the hospital environment \& volunteers and then identified them by standard methods. In case of hospital isolates, highest percentage of resistance was shown against Erythromycin (88.89\%) and Ampicillin (83.33\%). Significant resistance was also observed in cases of Ciprofloxacin $(33.33 \%)$ and Tetracycline $(33.33 \%)$. Lowest percentage of resistance was shown against Streptomycin $(11.11 \%)$ and Vancomycin shows only intermediate resistant pattern $(11.11 \%)$. On the other hand, community isolates were $100 \%$ sensitive against four antibiotics except Ampicillin (60\% resistant) and Vancomycin (20\% resistant). From the result it is easily discernable that, there are significant differences in the resistance pattern among hospital environment isolates and community acquired isolates. Samples were collected from different locations of hospital surgery room e.g. floor, wall, operating bed, trolley, sitting tool, cabinet etc. As anticipated, highest number of isolates showing resistance against these antibiotics were from the tool used for sitting.
\end{abstract}

\section{Keywords}

Staphylococcus aureus, Nosocomial Infection, Antibiotic Resistant

"Corresponding author.

How to cite this paper: Roy, P.C., Shaheduzzaman, M., Sultana, N. and Jahid, I.K. (2015) Comparative Antibiotic Sensitivity Pattern of Hospital and Community Acquired Staphylococcus aureus Isolates of Jessore, Bangladesh. Journal of Biosciences and Medicines, 3, 17-23. http://dx.doi.org/10.4236/jbm.2015.310003 


\section{Introduction}

Staphylococcus aureus is a ubiquitous commensal bacterium on human skins and anterior nares, but frequently causes severe infections in humans [1]. It is the most commonly isolated human bacterial pathogen and is an important cause of skin and soft-tissue infections, endovascular infections, pneumonia, septic arthritis, endocarditis, osteomyelitis, foreign-body infections and sepsis [2]. S. aureus is the prime pathogen of healthcare-associated infections which is called nosocomial infection. Perhaps, it is the pathogen of greatest concern that causes nosocomial infection because of its intrinsic virulence, ability to cause a diverse array of infections, capacity to adapt different environmental conditions and its nasal carriage which accounts for possible re-infection and also spread. Present years have seen an emergence of nosocomial infection with the introduction of new and sophisticated medical instruments. The incidence of nosocomial infections and the spectrum of pathogens specifically depend on the features of certain clinical wards and the existing/emerging predisposing factors. Nosocomial infections are caused even more frequently by multidrug-resistant bacteria that used to be isolated much more seldom previously but are significant regarding hospital hygiene.

The evolution of antimicrobial resistant bacterial species stems from factors like widespread and sometimes inappropriate use of antimicrobials, the extensive use of these agents as growth enhancers in animal feeds and the increase in regional and international travel with which antimicrobial-resistant bacteria cross geographical barriers [3]-[5]. S. aureus isolates from hospital across the world are increasingly resistant to a greater number of antimicrobial agents. As rapidly as new antibiotics are introduced, Staphylococci have developed efficient mechanisms to neutralize them. The difficult therapeutic problem of multidrug resistant $S$. aureus is just one example of the diminishing efficacy of antimicrobial agents for the treatment of bacterial infections. This trend is particularly alarming for $S$. aureus because of the severity and diversity of disease caused by this uniquely versatile pathogen. Methicillin-resistant Staphylococcus aureus (MRSA) infections are well-known worldwide as causing hospitalizations and deaths [6]. MRSA infections originated in hospitals [7] and then emerged into the community [8] [9].

As there are limited or no data available on antibiotic resistance pattern of S. aureus hospital isolates in Bangladesh, this study was undertaken. The strength and purpose of this study was to identify the health associated risks being faced in the present conditions of hospital. This study also focus on comparative antimicrobial resistance of community acquired and hospital acquired S. aureus isolates, which may help to create a baseline antibiotic resistance data and in formulating an effective infection control policy.

\section{Methodology}

\subsection{Sample Collection}

A total of 24 samples were collected from the Surgery Unit of Jessore-250 Bed General Hospital at Jessore district of Khulna, Bangladesh. It contains 2 (two) surgery rooms, each of which has 2 beds, several trolleys, 2 shelves and other instruments. Areas that were included in the study were as follows: Operating Bed surface, wall, floor, table for metal bowls, trolley for syringes and ampoules, sitting tool, metal table for keeping surgery instruments and wooden cabinet of documents. The first surgery unit room was selected as the other room was out of service at the time of the study. For community isolates, we collected Hand swab sample $(\mathrm{n}=12)$ from the students of different departments except Department of Microbiology of Jessore University of Science and Technology. The volunteer students who take any medication and went to hospital within 15 days were excluded from the study. Tests and other microbiological procedures were performed at the Laboratory of the Department of Microbiology, Jessore University of Science \& Technology. Samples were collected by rubbing saline soaked sterile cotton buds followed by two dry cotton bud rubbing on the designated surfaces. The swab collection was performed in an area of 25 sq. $\mathrm{cm}(5 \times 5 \mathrm{~cm})$ and from every site we collected triplicate sample. After collection, the swabs were streaked on 3 types of media: nutrient agar (NA), MacConkey agar and Mannitol Salt Agar and incubated for 24 hours at $37^{\circ} \mathrm{C}$. Distinct colonies from the selective media was subcultured into nonselective media and incubate as previously state condition. After overnight growth, colonies were stocked in glycerol broth for further analysis.

\subsection{Biochemical Identification}

Stocked isolates were revived in NA plates and used for biochemical tests. 
Catalase test: Two drops of $3 \%$ Hydrogen Peroxide were placed on a sterile glass slide. Then a single colony from each of the revived culture of the samples was mixed in a drop with a sterile nichrome wire loop. Another drop was left intact for control. Formation of bubble in the hydrogen peroxide drop indicates the positive result. Results for catalase test were noted down for each of the isolate.

Coagulase test: The tests were carried out by using human plasma. The suspected isolates were picked and inoculated into a sterile test tube with $5 \mathrm{ml}$ of Phosphate-Buffered Saline. The optical density (O.D.) of the bacterial suspension was measured by using spectrophotometer at $600 \mathrm{~nm}$. After that, $150 \mu \mathrm{l}$ of bacterial suspension and $350 \mu \mathrm{l}$ of human blood plasma were pipetted into sterile test tube and mixed well. The test tubes were incubated at $37^{\circ} \mathrm{C}$ for 4 hours and the clot formation was observed for every 30 minutes. For the negative samples, the test tubes were further incubated for 24 hours and observed for clot formation. For the positive results, the test tubes were re-incubated for overnight to observe the clot lyses.

\subsection{Antibiogram}

Antimicrobial susceptibility was determined by the disk diffusion method. Multiple colony from the pure culture of the isolate were picked by using sterile inoculating loop and inoculated into a sterile test tube with $5 \mathrm{ml}$ of Phosphate-Buffered Saline. The O.D. of the suspension was measured by spectrophotometer and should not exceed $0.5 \mathrm{McF}$ arland at $600 \mathrm{~nm}$ wavelength. Then, sterile cotton swabs were used to spread the growth evenly on the surface of Mueller-Hilton (MH) agar. Six different types of antibiotic disks were placed on the surface of the inoculated $\mathrm{MH}$ agar plates. The $\mathrm{MH}$ agar plates were incubated for 18 to 24 hours at $37^{\circ} \mathrm{C}$. The diameter of inhibition zone was measured and antibiogram was determined according to the Clinical and Laboratory Standard Institute (CLSI) guideline (Table 1).

\section{Result}

The areas in the surgery room that are most prone to come under human contact were targeted as these areas are most probable to find $S$. aureus. After isolation and biochemical tests, 125 isolates from the hospital and 45 isolates from the hand swab were identified as S. aureus. Antibiogram was performed by Kirby-Bauer's Disc Diffusion Method using 6 antibiotics: Vancomycin, Ampicillin, Ciprofloxacin, Erythromycin, Streptomycin and Tetracycline. Among the hospital isolates highest percentage of resistance showed against erythromycin (88.89\%) and lowest against streptomycin (11.11\%) (Figure 1). Vancomycin shows $11.11 \%$ intermediate resistant pattern where $88.89 \%$ isolates was sensitive against it. The other four antibiotic shows different sensitivity pattern, after erythromycin ampicillin is the second highest (83.33\%) followed by ciprofloxacin and tetracycline which account 33.33\% each (Figure 1). In case of hand swab isolates, all 45 isolates showed different types of antibiogram pattern. Among the six antibiotics these isolates shows resistance against two antibiotics. Highest percentage of resistance (60\%) was showed against ampicillin followed by vancomycin which show $20 \%$ resistance (Figure 2). Four antibiotics (ciprofloxacin, erythromycin, streptomycin and tetracycline) showed 100\% sensitivity in case of community acquired (hand swab) isolates.

In our study, we also observed that ampicillin was the only antibiotic which show resistance in both types of isolates. It should be noted that, vancomycin and streptomycin are more effective and erythromycin shows less

Table 1. CLSI standards of antibiotics inhibition zone diameter measurement.

\begin{tabular}{ccccc}
\hline \multirow{2}{*}{ Antimicrobial agent } & Disk content $(\mu \mathrm{g})$ & \multicolumn{3}{c}{ Diameter zone (mm) } \\
\cline { 3 - 5 } & 30 & $\mathrm{R}$ (resistant) & I (intermediate) & $\mathrm{S}$ (sensitive) \\
\hline Vancomycin & 2 & - & - & $\geq 15$ \\
Ampicillin & 5 & $\leq 26$ & $27-35$ & $\geq 36$ \\
Ciprofloxacin & 30 & $\leq 15$ & $16-20$ & $\geq 21$ \\
Erythromycin & 10 & $\leq 13$ & $14-22$ & $\geq 23$ \\
Streptomycin & 30 & $\leq 11$ & $12-14$ & $\geq 15$ \\
Tetracycline & & $\leq 14$ & $15-18$ & $\geq 19$ \\
\hline
\end{tabular}




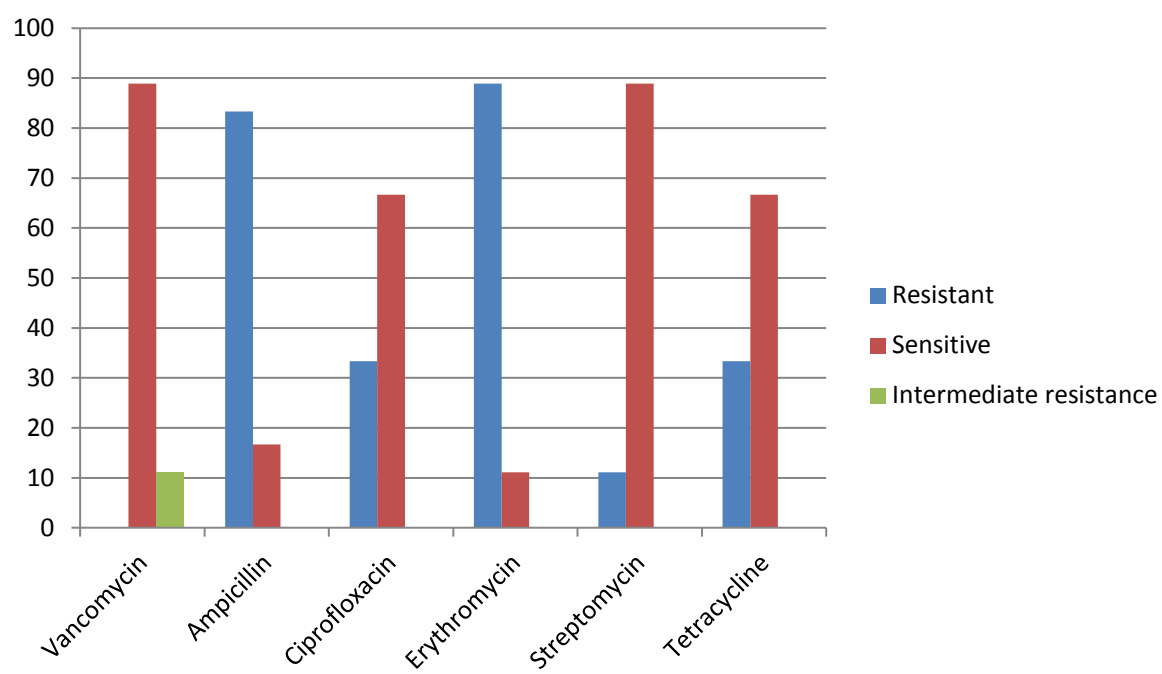

Figure 1. Antibiotic sensitivity pattern of hospital surgery unit isolates.

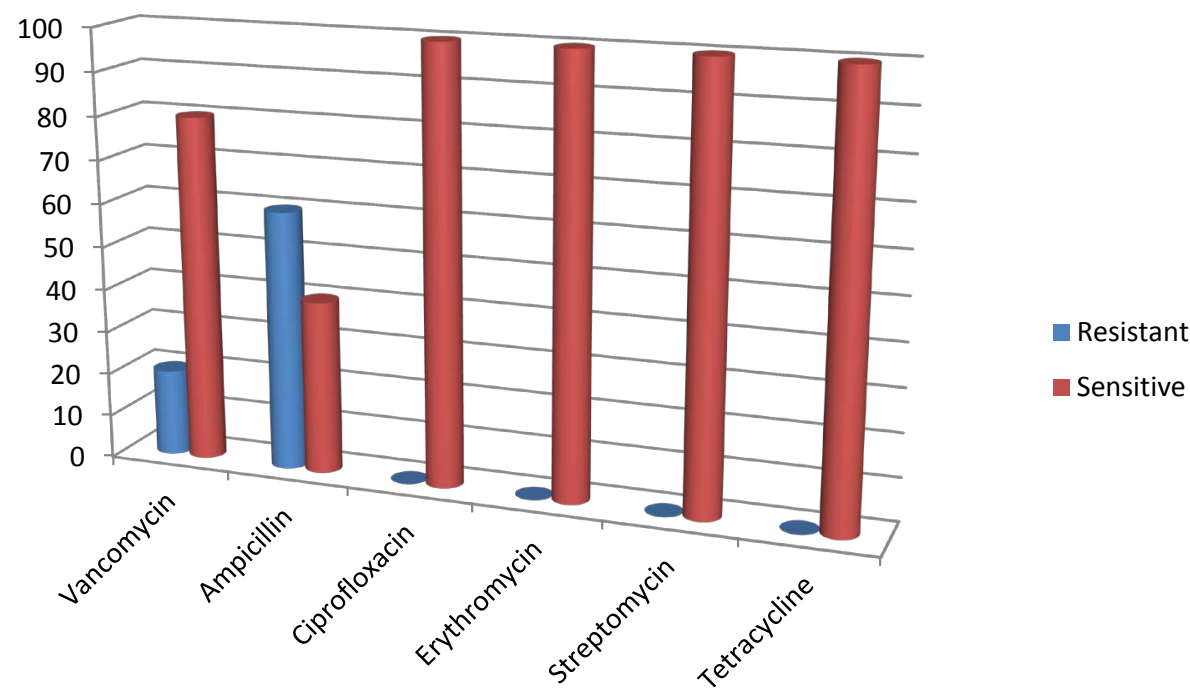

Figure 2. Antibiotic sensitivity pattern of community acquired (hand swab samples from volunteers) isolates.

effectiveness against hospital acquired isolates. Unlike hospital isolates, community isolates were more sensitive against four antibiotic and were less sensitive against ampicillin and vancomycin (Figure 3). The two types of isolates show completely opposite antibiotic sensitivity pattern where hospital isolates were more resistant and community isolates were more sensitive, which can be a significant finding in our experiments. In our study we also try to determine the distribution pattern of resistant bacteria in the hospital environment. Samples collected from every site showed multi-drug resistant strains of $S$. aureus. Almost all the isolates were resistant against more than one type of antibiotics (Table 2). Highest number of isolates showing resistance against these antibiotics was from the tool used for sitting. Same types of resistant bacteria obtained from cabinet for keeping documents and table for metal bowls, being resistant against 4 types of antibiotics and showing intermediate resistance against one.

\section{Discussion}

S. aureus is associated with a wide spectrum of infections ranging from mild skin and soft tissue infections to life threatening sepsis. The present study was conducted to know the common antibiotic resistance patterns in 


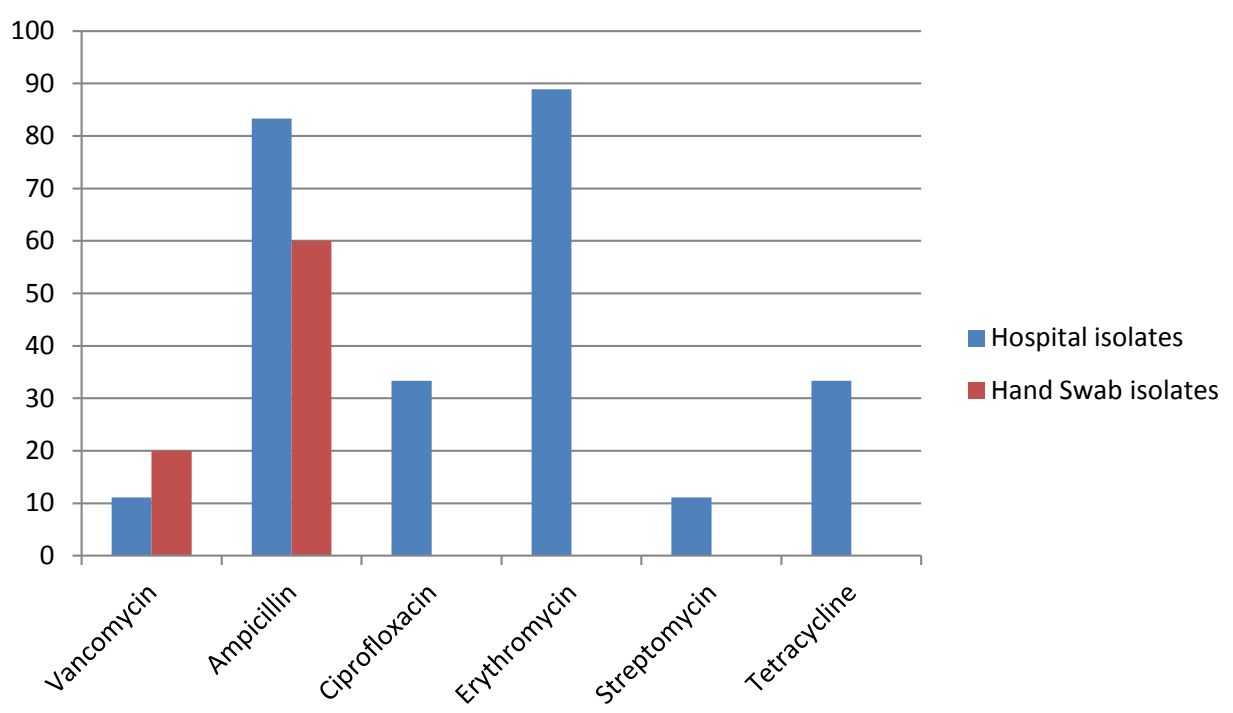

Figure 3. Comparative antibiotic resistance pattern of hospital acquired and community acquired isolates of S. aureus.

Table 2. Distribution pattern of antibiotic resistant bacteria in the hospital. Here, $\mathrm{A}=$ Operating bed, $\mathrm{B}=\mathrm{Wall}, \mathrm{C}=\mathrm{Floor}, \mathrm{D}$ = Table for metal bowls, $\mathrm{E}$ = Trolley for syringes and ampoules, $\mathrm{F}=$ Sitting tool, $\mathrm{G}=$ Metal table for keeping surgery instruments, $\mathrm{H}=$ Wooden cabinet for documents storage and $\mathrm{I}=$ Intermediate resistant. In case of antibiotic, $\mathrm{V}=\mathrm{Vancomycin}$, $\mathrm{A}=$ Ampicillin, $\mathrm{C}=$ Ciprofloxacin, $\mathrm{E}=$ Erythromycin, $\mathrm{S}=$ Streptomycin and $\mathrm{T}=$ Tetracycline. Yes denotes presence and No denotes absence of resistant bacteria.

\begin{tabular}{|c|c|c|c|c|c|c|}
\hline \multirow{2}{*}{ Location of isolates } & \multicolumn{6}{|c|}{ Types of antibiotic } \\
\hline & V resistant & A resistant & C resistant & E resistant & S resistant & T resistant \\
\hline A. & No & Yes & Yes & Yes & Yes & No \\
\hline B. & No & Yes & No & Yes & No & Yes \\
\hline C. & No & Yes & Yes & Yes & No & Yes \\
\hline D. & Yes (I) & Yes & Yes & Yes & No & Yes \\
\hline E. & No & Yes & No & Yes & No & No \\
\hline F. & No & Yes & Yes & Yes & Yes & Yes \\
\hline G. & No & Yes & No & Yes & No & No \\
\hline H. & Yes (I) & Yes & Yes & Yes & No & Yes \\
\hline
\end{tabular}

clinical isolates of S. aureus at the surgery unit of 250 Bed General Hospital, Jessore, Khulna, Bangladesh. This study was performed with a view to identifying the microorganisms that might carry the risk of infecting the patients being operated in the surgery units. Opportunistic pathogens generally do not pose any health risks, but during these operations the immune system of the patient's body remains in a suppressed state by the condition of the body and the application of various immune-suppressive drugs. For this reason, during this time the opportunistic pathogens residing in the patient's body, as well as microorganisms from the environment finds the chance to enter the patient's body and cause disease.

S. aureus remains the most prominent aetiology of pyogenic infections [10]. The antibiotics used in this study were vancomycin, ampicillin, ciprofloxacin, erythromycin, streptomycin and tetracycline. Except vancomycin, higher rate of resistance for all other antibiotics was shown in the surgery unit isolates. For vancomycin, the hospital isolates were intermediately resistant with a rate of $11.11 \%$. Highest rate of resistance was in case of erythromycin, which is $\mathbf{8 8 . 8 9 \%}$. Whereas in the case of isolates from the skin of volunteers, the highest rate of resistance was shown against ampicillin, which is $60 \%$. Resistance against ampicillin in hospital isolates was 
also pretty high, $83.33 \%$. It is clearly noticeable here that, the hospital surgery unit isolates were more resistant than the volunteers skin isolates.

Many factors may have contributed to such level of resistance, including inappropriate use of antibiotics by health professionals, unskilled practitioners and over the counter availability of antibiotics etc. In Bangladesh, it is a common practice that antibiotics can be purchased without prescription, which leads to misuse of antibiotics by the public, thus contributing to the emergence and spread of antimicrobial resistance in community also. Other causal factors could be poor hospital hygienic conditions, accounting for the spread of resistant bacteria and inadequate surveillance, i.e. lack of information from routine antimicrobial susceptibility testing of bacterial isolates and surveillance testing of bacterial isolates and surveillance of antibiotic resistance, all of which are crucial for good clinical practice and for rational policies against antibiotic resistance. In this study, vancomycin shows intermediate resistance $(11.11 \%)$ in case of the hospital isolates, conversely skin sample isolates show $20 \%$ resistance. Vancomycin retains an almost similar pattern of sensitivity for both hospital samples and skin samples in this study. Among six antibiotics vancomycin can be an ideal choice for the treatment of hospital acquired S. aureus infection as it shows more sensitivity pattern. Erythromycin was the least successful antibiotic against the hospital isolates in this study. A similar result was found for erythromycin in case of surgical site infection isolates by other researcher [11].

Samples for the hospital isolates were collected from various places of the hospital surgery room. The places included operating bed, wall, floor, wooden table in which metal boxes and bowls are kept, smaller trolley in which ampoules and other materials are kept, tools which are used for sitting, metal cabinet for keeping various operating apparatus and a wooden table in which various documents were kept. The places were selected with a view to identifying the places in which human interactions happen routinely, so that they have a higher probability of hosting microorganisms. The distribution pattern clearly demonstrate that everywhere of the operating room is occupied by multiple drug resistant isolates of $S$. aureus.

The prevalence of multidrug resistant $S$. aureus in the surgery unit of 250 Bed General Hospital, Jessore seemed to be high in this study. These results suggest that the resistance capacity of microorganisms against antibiotics is increasing day by day. Their emergence, antibiotic sensitivity pattern and other characteristics demand future endeavors in this field. The study covered resistance pattern only against six types of antibiotics, moreover, our prime concern was $S$. aureus. It can be clearly anticipated that other species of hospital acquired pathogenic/opportunistic microorganisms with the same or more severe resistance pattern like S. aureus have already emerged. They pose a large risk to the health care sector and relevant areas of Bangladesh. In our study we don't determine the Methicillin-resistant pattern of both isolates which is well-known worldwide as causing hospitalizations and deaths. It is suggested that future studies should include a broader range of antibiotics and a larger number of microbial spp. all over the country, so that we can get a clear idea about the distribution pattern of antibiotic resistant bacteria.

\section{Acknowledgements}

The author's are grateful to the director of Jessore-250 Bed General Hospital of Jessore, Khulna, Bangladesh for his cooperation during sample collection. We are also thankful to the students from whom we collected our community samples.

\section{References}

[1] Kluytmans, J., Van Belkum, A. and Verbrugh, H. (1997) Nasal Carriage of Staphylococcus aureus: Epidemiology, Underlying Mechanisms, and Associated Risks. Clinical Microbiology Reviews, 10, 505-520.

[2] Lowy, F.D. (1998) Staphylococcus aureus Infections. New England Journal of Medicine, 339, 520-532. http://dx.doi.org/10.1056/NEJM199808203390806

[3] Cohen, M.L. (1992) Epidemiology of Drug Resistance: Implications for a Post-Antimicrobial Era. Science, 257, 10501055. http://dx.doi.org/10.1126/science.257.5073.1050

[4] Tomasz, A. (1994) Multiple-Antibiotic-Resistant Pathogenic Bacteria-A Report on the Rockefeller University Workshop. New England Journal of Medicine, 330, 1247-1251. http://dx.doi.org/10.1056/NEJM199404283301725

[5] Swartz, M.N. (1997) Use of Antimicrobial Agents and Drug Resistance. The New England Journal of Medicine, 337, 491-492. http://dx.doi.org/10.1056/NEJM199708143370709 
[6] De Lencastre, H., Oliveira, D. and Tomasz, A. (2007) Antibiotic Resistant Staphylococcus aureus: A Paradigm of Adaptive Power. Current Opinion in Microbiology, 10, 428-435. http://dx.doi.org/10.1016/j.mib.2007.08.003

[7] Livermore, D.M. (2000) Antibiotic Resistance in Staphylococci. International Journal of Antimicrobial Agents, 16, 310. http://dx.doi.org/10.1016/S0924-8579(00)00299-5

[8] Naimi, T.S., LeDell, K.H., Como-Sabetti, K., Borchardt, S.M., Boxrud, D.J., et al. (2003) Comparison of Communityand Health Care-Associated Methicillin-Resistant Staphylococcus aureus Infection. JAMA, 290, 2976-2984. http://dx.doi.org/10.1001/jama.290.22.2976

[9] Kluytmans-VandenBergh, M. and Kluytmans, J. (2006) Community-Acquired Methicillin-Resistant Staphylococcus aureus: Current Perspectives. Clinical Microbiology and Infection, 12, 9-15. http://dx.doi.org/10.1111/j.1469-0691.2006.01341.x

[10] Adegoke, A.A. and Komolafe, A.O. (2009) Multi-Drug Resistant Staphylococcus aureus in Clinical Cases in Ile-Ife, Southwest Nigeria. International Journal of Medicine and Medical Sciences, 1, 068-072.

[11] Zoumalan, R.A. and Rosenberg, D.B. (2008) Methicillin-Resistant Staphylococcus aureus-Positive Surgical Site Infections in Face-Lift Surgery. Archives of Facial Plastic Surgery, 10, 116-123. http://dx.doi.org/10.1001/archfaci.10.2.116 